\title{
然
}

\section{LOS ACTIVOS FISCALES DIFERIDOS EN LA BANCA ESPAÑOLA}

A partir de 2014, la normativa internacional de solvencia, conocida como Basilea III, introdujo cambios para el cálculo de los recursos propios computables de la banca. Así, aquellos activos fiscales diferidos (DTA, según su acrónimo en inglés de Deferred Tax Assets) que dependen de la obtención de beneficios futuros para su recuperación dejarían gradualmente de computar para el cálculo del core capital (CET1), al considerarse que no está garantizado que mantengan su valor en caso de dificultades para la entidad. Para corregir esta situación y lograr que los DTA siguieran computando como capital de máxima calidad, algunos países europeos introdujeron cambios en su legislación del Impuesto sobre Sociedades, consistentes en permitir la monetización de una parte de estos activos fiscales diferidos y garantizar así su recuperación. El objetivo de este estudio es analizar las causas y la evolución de los cambios legales introducidos en España en 2013 y 2015 en relación con los DTA de la banca, así como sus implicaciones contables. El estudio concluirá analizando la situación actual y las perspectivas futuras.

Palabras clave: DTA, Basilea, ratios de capital, banca, provisiones, cambios legales.

Clasificación JEL: G28, H25, H32, H50, M41.

\section{Introducción}

Durante los años de la crisis, las provisiones realizadas por parte del sector financiero y la generación de bases imponibles negativas incrementaron considerablemente el stock de los DTA de la banca en algunos países europeos, entre ellos España.

Al depender la recuperación de estos DTA de la generación de beneficios fiscales futuros,

*Cuerpo Superior de Estadísticos del Estado. Ministerio de Economía, Industria y Competitividad.

**Profesor del Área de Economía Financiera y Contabilidad Universidad Complutense de Madrid.

Versión de octubre de 2017. la introducción de Basilea III $^{1}$ eliminaría ${ }^{2}$ la mayor parte de estos activos de los recursos propios, lo que llevaría a una reducción significativa de las ratios de capital de las entidades de crédito y a la necesidad de ampliar capital, posiblemente con nuevas inyecciones de dinero público.

Sin embargo, Basilea III contempla que los DTA puedan seguir computando como capital de máxima calidad si se convierten $\triangleright$

\footnotetext{
1 Por Basilea III se entiende la normativa internacional de solvencia constituida por CRR y CRD IV.

2 Aunque Basilea III concede un plazo de diez años para restar gradualmente los DTA, los mercados exigen que las entidades cumplan con los nuevos requisitos de solvencia desde el 1 de enero de 2014.
} 
en créditos fiscales exigibles ${ }^{3}$ frente a la Administración.

Tras la aprobación de las nuevas normas de solvencia, algunos países europeos (Italia, España, Portugal y Grecia) introdujeron modificaciones en su legislación del Impuesto sobre Sociedades, estableciendo las condiciones para la conversión de los DTA en créditos fiscales exigibles y evitar así que se redujeran las ratios de capital de la banca.

En nuestro país, el Real Decreto-ley 14/2013, de 29 de noviembre, introdujo el régimen de conversión de activos por impuesto diferido en la norma del Impuesto sobre Sociedades. Posteriormente, en 2015, para eliminar la incertidumbre sobre la compatibilidad de este instrumento con la legislación comunitaria, se modificó el tratamiento de los DTA mediante una enmienda en la Ley de Presupuestos Generales del Estado para 2016.

En este estudio se analizarán las causas, la evolución y las implicaciones contables de los cambios legales introducidos en España en 2013 y 2015 en relación con los DTA. Asimismo, se proporcionará un análisis del stock de los DTA acumulado por las principales entidades españolas durante el periodo 2011-2016.

\section{Definiciones}

\subsection{Los DTA según la normativa contable}

Los DTA están definidos en la Norma Internacional de Contabilidad (NIC) 12, Impuesto sobre las Ganancias, y en la Norma de valoración 13. a Impuestos sobre el Beneficio del Plan General de Contabilidad de España (PGCE). Se definen como cantidades de impuestos sobre

3 Artículo 39 de CRR. las ganancias (normalmente Impuesto sobre Sociedades) las que las sociedades podrán recuperar si generan ganancias fiscales (bases imponibles positivas) en ejercicios futuros.

Los DTA se originan como consecuencia de (i) diferencias temporarias deducibles; (ii) pérdidas obtenidas en ejercicios anteriores que aún no hayan sido objeto de deducción fiscal y (iii) créditos fiscales no utilizados procedentes de ejercicios anteriores.

Los activos por impuesto diferido se valorarán según los tipos de gravamen esperados en el momento de su reversión, según la normativa que esté vigente o aprobada y pendiente de publicación en la fecha de cierre del ejercicio, y de acuerdo con la forma en que racionalmente se prevea recuperar el activo.

\subsection{Diferencias temporarias}

Las diferencias temporarias son aquellas derivadas de la diferente valoración, contable y fiscal, atribuida a los activos, pasivos y determinados instrumentos de patrimonio propio de la empresa, en la medida en que tengan incidencia en la carga fiscal futura.

La valoración fiscal de un activo, pasivo o instrumento de patrimonio propio, denominada base fiscal, es el importe atribuido a dicho elemento de acuerdo con la legislación fiscal aplicable.

Las diferencias temporarias se originan normalmente por la existencia de desfases entre la base imponible y el resultado contable antes de impuestos, puesto que las normas contables que determinan el beneficio de una empresa (PGCE) pueden ser distintas de las normas fiscales (legislación del Impuesto sobre Sociedades) que determinan la base imponible. 
Así, cuando el resultado contable de una sociedad es menor que la base imponible, debido a diferencias temporales, la empresa paga un impuesto corriente superior al devengado contablemente. Para compensarlo, se genera un DTA ${ }^{4}$ a favor de la empresa que le permitirá reducir el impuesto sobre el beneficio a pagar en el futuro. Por el contrario, si el resultado contable es mayor que la base imponible, debido a diferencias temporales, se genera un pasivo por impuesto diferido que le obligará a pagar más impuesto sobre el beneficio en el futuro, que no es objeto de análisis en este estudio.

Un ejemplo significativo de diferencia temporaria es el deterioro para cubrir el riesgo de insolvencia (anteriormente denominado provisiones para insolvencias). Las pérdidas por deterioro de créditos se consideran un gasto que impacta el resultado contable. Sin embargo, a efectos fiscales, no se consideran un gasto deducible hasta que no se cumplan determinadas condiciones, lo que puede tener lugar en un periodo posterior al registro contable del deterioro. Estas dotaciones darán pues lugar a los DTA.

Otro ejemplo son las aportaciones a planes de pensiones para empleados, que afectan al resultado contable pero no a la base imponible hasta que el beneficiario cobra la prestación, dando lugar a la generación de los DTA.

Veamos un ejemplo numérico que da lugar al registro de activos por impuesto diferido. Una entidad al cierre del ejercicio $x$ tiene un saldo a cobrar de 10.000 u.m. sobre un cliente, del que estima que va recuperar un 50 por 100 , registrando el oportuno deterioro. Fiscalmente, ese deterioro no es deducible. El tipo de impuesto es del 30 por 100, sin que esté prevista

4 Calculado en función del impacto en la correspondiente partida del balance de la mencionada diferencia, que dará lugar a una diferencia temporaria deducible. una modificación en los próximos años. La diferencia entre la base imponible y el resultado contable es consecuencia de una diferencia temporal. Si se analiza el efecto en el valor del derecho, se produce una diferencia entre su valor contable y su base fiscal (valor fiscal) de 5.000 u.m., la diferencia temporaria deducible generada. Al aplicar el tipo impositivo a la diferencia temporaria deducible se obtendrá el DTA a favor de la entidad (1.500 u.m.).

CUADRO 1

EJEMPLO DE DIFERENCIAS TEMPORARIAS

\begin{tabular}{|l|r|r|}
\hline \multicolumn{1}{|c|}{ Ejercicio $x$} & Contable & \multicolumn{1}{|c|}{ Fiscal } \\
\hline Importe del derecho & 10.000 & 10.000 \\
Deterioro & -5.000 & 0 \\
Valor contable & $5.000(1)$ & \\
Base fiscal (valor fiscal) & & $10.000(2)$ \\
$\begin{array}{l}\text { Diferencia temporaria } \\
\text { (DT) }=(2)-(1)\end{array}$ & 5.000 \\
$\begin{array}{l}\text { Activo por impuesto } \\
\text { diferido (DTA) }\end{array}$ & $1.500=5.000$ * $30 \%$ \\
\hline Fuente: elaboración propia. & \\
\hline
\end{tabular}

\subsection{Pérdidas de ejercicios anteriores}

Asimismo, las pérdidas de ejercicios anteriores que no hayan sido objeto de deducción fiscal dan lugar a la aparición de los DTA.

En general, cuando una empresa tiene pérdidas materializadas, aparte de no pagar impuestos en el periodo, puede utilizarlas para reducir los impuestos que pagará en el futuro cuando vuelva a beneficios; es lo que se denomina bases imponibles negativas (BIN).

\subsection{Legislación nacional}

Si bien la definición de DTA es estándar, a nivel nacional, las condiciones para la generación y materialización de los DTA se $\triangleright$ 
establecen en la legislación del Impuesto sobre Sociedades, lo que da lugar a diferencias significativas entre países.

Por ejemplo, en España hay un plazo límite de 18 años para recuperar los DTA y, si no se logran compensar en ese período, se pierden. En otros países, sin embargo, esta limitación no existe.

También en algunos países está permitido el llamado carry back, aunque no es el caso en España. Si una empresa ha incurrido en pérdidas en un ejercicio, tras haber tenido beneficios y pagado los impuestos correspondientes en los años anteriores, se genera un derecho inmediato contra esos impuestos pasados, en la forma de una devolución en efectivo o la posibilidad de compensar impuestos a pagar en el futuro.

\subsection{DTA vs créditos fiscales}

En general, los DTA presentes en los balances de las empresas no pueden considerarse automáticamente créditos fiscales.

Los créditos fiscales son cantidades que pueden establecerse con certeza y no están sujetos a revisiones. No están basados en hipótesis, sino en hechos observables.

Por su parte, el cálculo del stock de los DTA se basa en distintas hipótesis (por ejemplo, sobre tipos impositivos y beneficios futuros) y está sujeto a revisiones continuas. Estos rasgos no parecen encajar con las características de los créditos fiscales.

Los DTA dan derecho a pagar menos impuestos en el futuro si se dan ciertas circunstancias; son activos que se realizarán si se cumplen determinadas condiciones. Sin embargo, un DTA no será "exigible" hasta que el derecho a pagar menos impuestos se materialice y se pueda establecer una cantidad determinada.

Así, algunos DTA puede que nunca se recuperen. Sin embargo, otros sí serán recuperados en algún momento, ya sea porque (i) las empresas generen bases imponibles positivas en el futuro o (ii) porque se hayan aprobado leyes que permiten la conversión de los DTA en créditos exigibles, pese a tener bases imponibles negativas.

\section{Cambios normativos recientes en España. Causas y evolución}

\subsection{Antecedentes}

Del apartado anterior (legislación nacional) puede deducirse que la normativa del Impuesto sobre Sociedades en España era, hasta hace poco, más restrictiva que en otros países europeos, como Alemania, Francia, Irlanda o Bélgica, lo que perjudicaría a las entidades españolas al entrar en vigor Basilea III.

Por otro lado, a raíz de la crisis financiera, la banca española había acumulado un importante stock de DTA, que superaba el 5 por 100 del PIB en 2013. Las razones para la generación de este stock fueron las siguientes, por orden de importancia:

1. La aprobación de dos Reales Decretoley en $2012^{5}$ obligó a todas las entidades de crédito a provisionar ingentes cantidades que provocaron pérdidas contables generalizadas en el sector, no deducibles fiscalmente, es decir, diferencias temporarias que generaron enormes DTA para el futuro.

5 Real Decreto-ley 2/2012, de 3 de febrero, de saneamiento del sector financiero, y Real Decreto-ley 18/2012, de 11 de mayo, sobre saneamiento y venta de los activos inmobiliarios del sector financiero. 
2. Por otro lado, las entidades rescatadas $^{6}$ y aquellas que absorbieron cajas intervenidas ${ }^{7}$ o insolventes aunque no llegaran a nacionalizarse ${ }^{8}$ tuvieron fuertes pérdidas derivadas de los grandes saneamientos acometidos, por lo que generaron bases imponibles negativas.

3. Finalmente, los dos grandes bancos españoles, Santander y BBVA, tienen fuertes compromisos por pensiones, algo que también afecta en menor medida a la banca mediana, dando lugar a diferencias temporarias que generaron unos DTA adicionales.

\subsection{Real Decreto-ley 14/2013, de 29 de noviembre}

Teniendo en cuenta el carácter restrictivo de la legislación española para la generación y utilización de los DTA en relación con otros países, así como el importante stock acumulado por las entidades financieras españolas, en noviembre de 2013, el Gobierno aprobó un Real Decreto-ley ${ }^{9}$ que eliminaba los inconvenientes introducidos por Basilea III, de forma que los DTA pudieran seguir computando en el capital y las entidades de crédito españolas pudiesen operar en un entorno competitivo más homogéneo y evitar tener que ampliar capital.

El mecanismo de conversión de los DTA en crédito exigible frente a la Administración tributaria se introduce en la disposición adicional vigésima segunda de dicho decreto-ley,

\footnotetext{
6 Bankia, NCG Banco, Catalunya Banc y Ceiss.

CAM en el caso de Sabadell, Unnim en el de BBVA, CCM en Liberbank y Banco de Valencia en CaixaBank.

8 Banca Cívica en el caso de CaixaBank y Pastor en el de Popular.

9 Real Decreto-ley 14/2013, de 29 de noviembre, de medidas urgentes para la adaptación del derecho español a la normativa de la Unión Europea en materia de supervisión y solvencia de entidades financieras.
}

que constituye una modificación de la Ley del Impuesto sobre Sociedades. Este mecanismo de conversión de DTA en créditos fiscales se conoce como monetización. Consiste en determinadas medidas destinadas a permitir que ciertos DTA puedan seguir computando como capital, en línea con la regulación vigente en otros Estados de la Unión Europea.

La introducción de este mecanismo supone que los DTA generados por provisiones y gastos de pensiones se recuperarán siempre, aunque no se prevean beneficios suficientes en el futuro e incluso en caso de quiebra. Si una entidad tiene pérdidas en el futuro, el Estado tendrá que poner parte del dinero pendiente de compensar para absorberlas, y si quiebra, el Estado aportará todo para intentar paliar la situación. Además, desaparece la limitación temporal para recuperar los DTA anteriores: si una entidad no los ha recuperado en 18 años, cobrará lo que le falte en el año 19. En definitiva, los DTA monetizables pasan a estar garantizados.

Es importante mencionar que la monetización es aplicable a todas las entidades sujetas al Impuesto sobre Sociedades y no solo a las instituciones financieras. Sin embargo, en la práctica, el sector bancario será el principal beneficiario, puesto que el stock de los DTA generado por las sociedades no financieras por provisiones o pensiones no es tan significativo.

En el caso concreto de las entidades de crédito, esta norma fortalece su solvencia al evitar que el régimen fiscal pueda crear distorsiones -que no existen en otras jurisdicciones- en el cálculo del capital regulatorio de las entidades financieras.

A continuación se resume la parte más relevante de este decreto ley. 
Conversión de los DTA en crédito exigible frente a la Administración tributaria

Los activos por impuesto diferido correspondientes a dotaciones por deterioro de los créditos $u$ otros activos derivados de las posibles insolvencias de los deudores no vinculados con el sujeto pasivo, así como los correspondientes a dotaciones o aportaciones a sistemas de previsión social y, en su caso, prejubilación, se convertirán en un crédito exigible frente a la Administración tributaria cuando se dé cualquiera de las siguientes circunstancias:

a) Que el sujeto pasivo registre pérdidas contables en sus cuentas anuales, auditadas y aprobadas por el órgano correspondiente.

b) Que la entidad sea objeto de liquidación o insolvencia judicialmente declarada.

\subsection{Consecuencias}

La monetización prevista en el anterior Real Decreto-ley (RDL) se aplica a los DTA generados por provisiones y por gastos de pensiones, que representan alrededor de un 60 por100 del stock total de los DTA. Sin embargo, la conversión en crédito fiscal no se aplica a los DTA generados a partir de bases imponibles negativas (BIN), que representan el 20 por 100 restante.

Así, a pesar de la aprobación del RDL, una cantidad importante de DTA (alrededor de 20.000 millones) deberá restarse de las ratios de solvencia en cualquier caso. Esto afecta principalmente a las entidades nacionalizadas, que son las que acumulan más BIN.

En abril del 2015, la Comisión Europea (CE) abrió una investigación para determinar si los avales otorgados por el sector público a la banca para poder computar ciertos DTA como capital eran, o no, una ayuda de Estado incompatible con la legislación comunitaria.

En septiembre de 2015, para eliminar las dudas sobre si el mecanismo de conversión de los DTA introducido en el RDL 14/2013 constituía o no ayuda de Estado, el Gobierno español propuso una serie de modificaciones en el tratamiento fiscal de los DTA en el Impuesto sobre Sociedades, tramitado como una enmienda en la Ley de Presupuestos Generales del Estado para 2016. La CE dio por buenas dichas modificaciones, que se aprobaron en octubre, quedando cerrada la investigación. Las modificaciones introducidas se explican en el siguiente apartado.

\subsection{Ley 48/2015, de 29 de octubre, de Presupuestos Generales del Estado para el año 2016}

Las modificaciones en el tratamiento de los DTA aparecen en el artículo 65 de la Sección Segunda, correspondiente al Impuesto Sobre Sociedades.

Se incide en que la monetización consiste en un derecho opcional a favor del contribuyente.

Se establecen tratamientos diferentes para los DTA generados antes y después del 1 de enero de 2016, consistentes en lo siguiente:

1. Los DTA generados antes del 1 de enero de 2016, que estuvieran cubiertos por la garantía recogida en el RDL 14/2013, la mantendrán en el futuro. No obstante, si los beneficiarios de la garantía hubieran pagado menos impuestos, entre los años 2008 y 2015, que el valor de esos DTA garantizados, deberán abonar una prestación patrimonial anual en favor del Estado del 1,5 por 100 de la diferencia entre ambos importes.

2. A partir del ejercicio 2016 sólo se garantizarán aquellos DTA que cumplan los mismos requisitos que en la norma de 2013 pero con un límite que depende del Impuesto sobre Sociedades pagado. Es decir, no se podrán generar DTA garantizables en aquellas entidades que no $D$ 
hayan generado cuotas líquidas positivas por el citado impuesto.

La prestación patrimonial introducida en esta ley tiene como objeto «compensar el riesgo que se transfiere al Estado» por la monetización de los DTA.

Además, la aplicación del derecho de monetización conllevará que las entidades deban incluir determinada información en la declaración del Impuesto sobre Sociedades: importe total de los DTA, importe total y año de generación de los DTA respecto de los que se tiene derecho a la conversión e importe total y año de generación de los DTA respecto de los que no se tiene derecho a la conversión.

\section{DTA en la banca española}

En el Cuadro 2 se presenta el stock de los DTA de las principales entidades financieras españolas para el periodo 2011-2016. Los datos se han obtenido de sus cuentas anuales consolidadas. Por esta razón, y sobre todo para entidades grandes, el stock incluye los DTA susceptibles de recuperarse en otros países (por ejemplo, los DTA de filiales en Portugal o Reino Unido). Así, al utilizarse las cuentas consolidadas, las cifras presentadas en el cuadro serán algo superiores a las citadas en la prensa en concepto de DTA de la banca española (estas últimas, en el entorno de los 50.000 millones en 2013).

A partir de 2013, año en que se aprobó el Real Decreto-ley, se proporciona una columna con el stock de los DTA monetizables, es decir, convertibles en créditos fiscales exigibles. La diferencia entre el stock de DTA y el stock de DTA monetizable constituye el stock de DTA generado principalmente por bases imponibles negativas, que podrá recuperarse solo en el caso de beneficios fiscales futuros.

En 2012 y, en menor medida, en 2013, se observa un aumento significativo del stock de DTA como consecuencia de los dos reales $\triangleright$

CUADRO 2 STOCK DE LOS DTA DE LAS PRINCIPALES ENTIDADES FINANCIERAS ESPAÑOLAS (2011-2016) (En millones de euros)

\begin{tabular}{|c|c|c|c|c|c|c|c|c|c|c|}
\hline \multirow{2}{*}{$\begin{array}{l}\text { Principales } \\
\text { entidades }\end{array}$} & \multirow{2}{*}{$\begin{array}{l}2011 \\
\text { DTA }\end{array}$} & \multirow{2}{*}{$\begin{array}{l}2012 \\
\text { DTA }\end{array}$} & \multicolumn{2}{|r|}{2013} & \multicolumn{2}{|r|}{2014} & \multicolumn{2}{|c|}{2015} & \multicolumn{2}{|r|}{2016} \\
\hline & & & DTA & $\begin{array}{c}\text { DTA } \\
\text { monetizable }\end{array}$ & DTA & $\begin{array}{c}\text { DTA } \\
\text { monetizable }\end{array}$ & DTA & $\begin{array}{c}\text { DTA } \\
\text { monetizable }\end{array}$ & DTA & $\begin{array}{c}\text { DTA } \\
\text { monetizable }\end{array}$ \\
\hline Bankia & 5.568 & 8.909 & 8.628 & 5.249 & 8.351 & 5.521 & 8.082 & 5.484 & 7.963 & 5.515 \\
\hline Bakinter & 103 & 148 & 132 & 89 & 144 & 86 & 147 & 90 & 165 & 68 \\
\hline BBVA (1) & 6.332 & 9.871 & 9.202 & 4.373 & 10.391 & 4.881 & 15.878 & 9.536 & 16.391 & 9.431 \\
\hline CaixaBank (2) & 761 & 6.295 & 9.584 & 4.721 & 9.390 & 4.721 & 10.093 & 5.586 & 9.642 & 5.802 \\
\hline Catalunya Banc & 1.968 & 1.626 & 3.516 & 3.482 & 3.562 & 3.466 & - & - & - & - \\
\hline Liberbank & 1.044 & 1.815 & 1801 & 1.123 & 1.808 & 1.156 & 1.864 & 1.197 & 1.763 & 1.209 \\
\hline Popular & 1.041 & 3.527 & 3.143 & 1.768 & 3.436 & 1.738 & 3.444 & 2.058 & 4.907 & 2.037 \\
\hline Sabadell & 997 & 5.609 & 6.216 & 4.790 & 6.144 & 5.283 & 6.576 & 5.521 & 6.775 & 5.806 \\
\hline Santander & 17.761 & 19.757 & 21.068 & 7.902 & 22.164 & 8.444 & 22.045 & 8.887 & 21.264 & 9.469 \\
\hline Totales & 35.575 & 57.557 & 63.290 & 33.497 & 65.390 & 35.296 & 68.129 & 38.359 & 68.870 & 39.337 \\
\hline \multicolumn{11}{|c|}{ (2) El importe del ejercicio 2013 se ha estimado en función de 2014 por no aparecer en la memoria. } \\
\hline Eunto olahoraci & incos & in & ato & eolidadaen $d x$ & endifore $>$ & tesentidades & & & & \\
\hline
\end{tabular}


decretos que obligaron a las entidades de crédito a provisionar cantidades importantes. A partir de 2013, el stock de DTA ralentiza su crecimiento, hasta prácticamente estabilizarse en 2016. Con la aprobación de la Ley de Presupuestos de 2016, se espera un crecimiento del stock de DTA monetizables moderado a partir de 2016.

Las entidades con mayor cantidad de DTA son Santander y BBVA. En ambos casos se observa una proporción elevada de DTA no monetizable, consecuencia de la absorción de entidades que habían registrado bases imponibles negativas.

\section{Tratamiento de los DTA en contabilidad nacional e impacto en las cuentas de las Administraciones Públicas (AAPP)}

\subsection{Normas}

Una de las diferencias entre la contabilidad empresarial y la contabilidad nacional es que la primera admite el registro de créditos fiscales sin estar reconocida de forma expresa la exigibilidad a las AAPP. Sin embargo, este no es el caso en la contabilidad nacional.

Así, en la contabilidad empresarial, los DTA aparecen como activos en el balance de las sociedades, mientras que en contabilidad nacional no se procede al registro de estos activos, al considerarlos contingentes.

En contabilidad nacional, los DTA se registrarán únicamente una vez que den lugar a créditos fiscales exigibles, y en ese caso se aplicarán las reglas definidas en el Sistema Europeo de Cuentas (SEC 2010), que establece registros distintos para los créditos fiscales pagaderos y los no pagaderos.
Como se vio en el apartado 2.5, los DTA darán lugar a créditos fiscales exigibles a las AAPP cuando (i) las empresas generen bases imponibles positivas en el futuro o cuando (ii) se moneticen cantidades de DTA, ya sea por la ocurrencia de pérdidas o por liquidación.

Los créditos fiscales no pagaderos se registran como un ingreso negativo para las AAPP en el momento en que el crédito fiscal se utiliza para pagar menos impuestos. Un ejemplo de crédito fiscal no pagadero ocurre cuando una empresa genera beneficio fiscal y revierten las diferencias temporarias pasadas, permitiendo la recuperación de ciertos DTA.

Por el contrario, los créditos fiscales pagaderos se registran como gasto y por su importe total en el momento en que la Administración reconoce el derecho del contribuyente a pagar menos impuestos, independientemente de que la recuperación del importe se realice en un periodo futuro. Un ejemplo de crédito fiscal pagadero serían los DTA que se moneticen siguiendo el RDL de noviembre de 2013.

Ambos tipos de crédito fiscal impactan al déficit negativamente. La diferencia principal entre ambos reside en el momento del registro y en los importes a contabilizar: (i) a medida que las cantidades se recuperan, en el caso de los no pagaderos; y (ii) por el importe total en el momento en que las AAPP reconocen una obligación en el caso de los pagaderos.

Por último, las cantidades percibidas por las AAPP en concepto de prestación patrimonial se registrarán como un ingreso en el momento en que se presente la declaración del impuesto.

\subsection{Impacto hasta la fecha}

Si bien el stock de DTA monetizable en los balances de la banca es considerable (en $\triangleright$ 
el entorno de los 30.000 millones en España), lo cierto es que hasta el final de 2016 no se han monetizado cantidades significativas. Por tanto, hasta finales de 2016, el impacto del régimen de conversión de los DTA en contabilidad nacional ha sido prácticamente nulo.

Esta situación podría cambiar en 2017, puesto que el Banco Santander tendría derecho a reclamar alrededor de 500 millones en efectivo ante la Agencia Tributaria, consecuencia de las pérdidas millonarias que sufrió el Popular en el año 2016 y que dan derecho a la monetización de esa cantidad. En el caso de efectuarse la monetización, se trataría de un crédito fiscal pagadero y la cantidad total se imputaría como gasto de 2017, con impacto en el déficit público de dicho año.

Sin embargo, las operaciones y las cantidades anteriores no podrán confirmarse hasta que no se hagan públicas las operaciones no financieras de las AAPP a lo largo de 2017 y los datos detallados de la Agencia Tributaria.

Por lo que respecta a la prestación patrimonial que la banca debe pagar para mantener sus DTA, según los datos de ejecución presupuestaria publicados por la IGAE, se estima que dicha prestación ha aportado unos ingresos de 471 millones al Estado en septiembre de 2017. Esta cantidad impactará positivamente el déficit de las AAPP en 2017.

\section{Conclusión}

A raíz de la aprobación de Basilea III, en España se tomaron una serie de medidas con el objeto de que las entidades de crédito españolas pudiesen operar en un entorno competitivo homogéneo y se evitase, en la medida de lo posible, recurrir a nuevas ampliaciones de capital.

Dichas medidas consistieron en sendos cambios legislativos del Impuesto sobre Sociedades, que fueron aceptados por la Comisión Europea tras las modificaciones introducidas en 2015.

Estas medidas permiten la monetización (y el cómputo como capital de máxima calidad) del 60 por 100 del stock total de DTA. Sin embargo, el resto del stock, generado por la existencia de BIN negativas en periodos pasados, no es monetizable y deberá, por tanto, deducirse del cálculo de los recursos propios.

La aprobación de la Ley de Presupuestos Generales del Estado para 2016 solucionó la situación para los DTA existentes antes de 2016, pero dificulta la generación de los mismos en lo sucesivo.

Finalmente, cabe destacar que el impacto de estos cambios legales en las cuentas de las AAPP desde una óptica de contabilidad nacional fue muy limitado hasta 2016. No obstante, las cifras de 2017 podrían verse impactadas tanto por el lado del gasto (monetización de los DTA del Popular por haber notificado pérdidas en 2016) como por el lado del ingreso (prestación patrimonial a pagar por la banca).

Finalmente, ha de comentarse que las fórmulas aprobadas en otros países periféricos, como Italia, Portugal y Grecia, tienen en común con el mecanismo español la posibilidad de monetizar ciertos DTA, permitiendo que estos computen para las ratios de capital. No obstante, se aprecian diferencias significativas con la legislación y el mecanismo aprobado en España.

\section{Bibliografía}

[1] BANCO DE ESPAÑA (2013). Nota informativa sobre tratamiento fiscal de activos fiscales diferidos (03.12.2013).

[2] EUROSTAT. COMISIÓN EUROPEA (2010). Sistema de Cuentas Nacionales y Regionales, SEC 2010. 
[3] EUROSTAT. COMISIÓN EUROPEA (2010). Eurostat Guidance Note. 29 August 2014. Deferred tax assets (DTAs) and recording of tax credits related to DTAs in ESA 2010.

[4] EUROSTAT. COMISIÓN EUROPEA (2016). Manual on Government Deficit and Debt (MGDD).

[5] LEY 48/2015, de 29 de octubre, de Presupuestos Generales del Estado para el año 2016. España.

[6] MINISTERIO DE ECONOMÍA Y COMPETITIVIDAD. Nota de prensa de 28 de septiembre de 2015.
[7] REAL DECRETO-LEY 14/2013, de 29 de noviembre, de medidas urgentes para la adaptación del derecho español a la normativa de la Unión Europea en materia de supervisión y solvencia de entidades financieras. España.

[8] REGULATION(EU)No575/2013OFTHEEUROPEAN PARLIAMENT AND OF THE COUNCIL of 26 June 2013 on prudential requirements for credit institutions and investment firms and amending Regulation (EU) No 648/2012. 